\title{
A SCHRÖDER-BERNSTEIN THEOREM IN BAER*-RINGS WITH LATTICE-THEORETIC PROOF
}

\author{
JOSUKE HAKEDA
}

\begin{abstract}
The Schröder-Bernstein theorem (SB-theorem) for *-equivalence of projections of a Baer*-ring is known.

Here, we will prove an SB-theorem for algebraic equivalence (Theorem A) as a consequence of a lattice-theoretic SB-theorem (Theorem B). Theorem A and the known result about *-equivalence will be derived from Theorem B.
\end{abstract}

An element $e$ of an involutive ring $A$ is called a projection if it is idempotent $\left(e^{2}=e\right)$ and selfadjoint $\left(e^{*}=e\right)$. The relation $e=e f$ defines a partial ordering of projections, denoted $e \leqslant f$. Projections $e, f$ of $A$ are said to be algebraically equivalent if there exist $x, y$ in $A$ such that $y x=e$ and $x y=f$, and *-equivalent if there exists $x$ in $A$ such that $x^{*} x=e$ and $x x^{*}=f$. We write $e \sim f$ for either of these relations, indicating in the context which of the relations is intended. The notation $e \lesssim f$ means that $e \sim f_{0}$ for some projection $f_{0} \leqslant f$.

An involutive ring $A$ is called a Baer*-ring if, for every subset $S$ of $A$, the right annihilator of $S$ has the form $e A, e$ a projection [3].

Theorem A. Let $e, f$ be projections in a Baer*-ring $A$. If $e \lesssim f$ and $f \lesssim e$ for algebraic equivalence, then $e \sim f$.

The analogue of Theorem A for *-equivalence is known [4, Theorem]. We shall give a lattice-theoretic proof of Theorem A.

Definition. An orthocomplemented lattice $L$ is called orthomodular if its orthocomplementation $x \mapsto \theta(x)(x \in L)$ satisfies the identity

$$
b=a \vee(b \wedge \theta(a))
$$

for $a \leqslant b(a, b \in L)$

LEMMA [5, Lemma 29.15]. Let $L$ be an orthomodular lattice with orthocomplementation $x \mapsto \theta(x)(x \in L)$. For $a \in L,[0, a]=\{x \in L: x \leqslant a\}$ is an orthomodular lattice for the orthocomplementation

$$
x \mapsto \theta_{a}(x)=a \wedge \theta(x)
$$

for $x \in[0, a]$. If $a \leqslant \theta(b)$, we write $a \perp b$.

Received by the editors March 28, 1978 and, in revised form, August 7, 1978.

AMS (MOS) subject classifications (1970). Primary 46K99, 46L99, 06A25, 06A23; Secondary 16A28, 16A32.

Key words and phrases. Schröder-Bernstein theorem, Baer*-ring, orthomodular lattice, complete lattice. 
THEOREM B. Suppose the orthomodular lattice $L$ is complete and possesses an equivalence relation $\sim$ satisfying the following conditions: (1) if $a_{1} \perp a_{2}, b_{1} \perp b_{2}$ and $a_{i} \sim b_{i}(i=1,2)$, then $a_{1} \vee a_{2} \sim b_{1} \bigvee b_{2}$;

(2) If $a \lesssim b$, then there exists an order-preserving mapping $\varphi$ of $[0, a]$ into $[0, b]$ such that $x \sim \varphi(x)$ for all $x \in[0, a]$.

Then $a \lesssim b$ and $b \lesssim a$ imply $a \sim b$ for $a, b \in L$.

Proof. From condition (2), there exist order-preserving mappings $\varphi_{1}$ and $\varphi_{2}$ such that $\varphi_{1}$ msps $[0, a]$ into $[0, b]$ and $\varphi_{2}$ maps $[0, b]$ into $[0, a]$. Define a mapping $\varphi$ of $[0, b]$ into $[0, b]$ by the formula

$$
\varphi=\theta_{b} \circ \varphi_{1} \circ \theta_{a} \circ \varphi_{2} \text {. }
$$

By the lemma, $\varphi$ is an order-preserving mapping in the complete lattice $[0, b]$; therefore, by [7, Theorem 1], there eists $c_{0} \in[0, b]$ such that $\varphi\left(c_{0}\right)=c_{0}$. Since $\theta_{b} \circ \theta_{b}=1$,

$$
\theta_{b}\left(c_{0}\right)=\theta_{b}\left(\varphi\left(c_{0}\right)\right)=\varphi_{1}\left(\theta_{a}\left(\varphi_{2}\left(c_{0}\right)\right)\right) .
$$

By condition (2), one has

$$
\theta_{b}\left(c_{0}\right)=\varphi_{1}\left(\theta_{a}\left(\varphi_{2}\left(c_{0}\right)\right)\right) \sim \theta_{a}\left(\varphi_{2}\left(c_{0}\right)\right)
$$

and $c_{0} \sim \varphi_{2}\left(c_{0}\right)$. Therefore, by the condition (1),

$$
b=c_{0} \vee \theta_{b}\left(c_{0}\right) \sim \varphi_{2}\left(c_{0}\right) \vee \theta_{a}\left(\varphi_{2}\left(c_{0}\right)\right)=a \text {. Q.E.D. }
$$

Proof of THEOREM A. The projections of $A$ form a complete orthomodular lattice with the orthocomplementation $e \mapsto 1-e$. Algebraic equivalence satisfies condition (1) [3, Theorem 17], and condition (2) as a special case of [6, Lemma 2.2]. Quote Theorem B. Q.E.D.

Remark 1. *-equivalence satisfies condition (1) [3, Theorem 25] and condition (2) [3, Theorem 24]. Therefore the SB-theorem for *-equivalence [4, Theorem] holds by Theorem B.

REMARK 2. By the order of set inclusion and orthocomplementation of usual complement, the family of all subsets of a set forms a complete orthomodular lattice. With slight modification, Theorem B gives a proof of the Bernstein theorem of set theory.

The author thanks Professor S. Maeda for his kind advice.

\section{REFERENCES}

1. S. K. Berberian, On the projection geometry of a finite $A W^{*}$-algebra, Trans. Amer. Math. Soc. 83 (1956), 493-508.

2. __ Baer*-rings, Springer-Verlag, Berlin, 1972.

3. I. Kaplansky, Rings of operators, Benjamin, New York, 1968.

4. A. Lebow, A Schroder-Bernstein theorem for projections, Proc. Amer. Math. Soc. 19 (1968), $144-145$.

5. F. Maeda and S. Maeda, Theory of symmetric lattices, Springer-Verlag, Berlin, 1970.

6. S. Maeda, On the lattice of projections of a Baer*-ring, J. Sci. Hiroshima Univ. Ser. A 22 (1958), 75-88.

7. A. Tarsky, A lattice-theoretical fixpoint theorem and its applications, Pacific J. Math. 5 (1955), 285-309.

Department of Basic Technology, Yamagata University, Yonezawa, Japan 